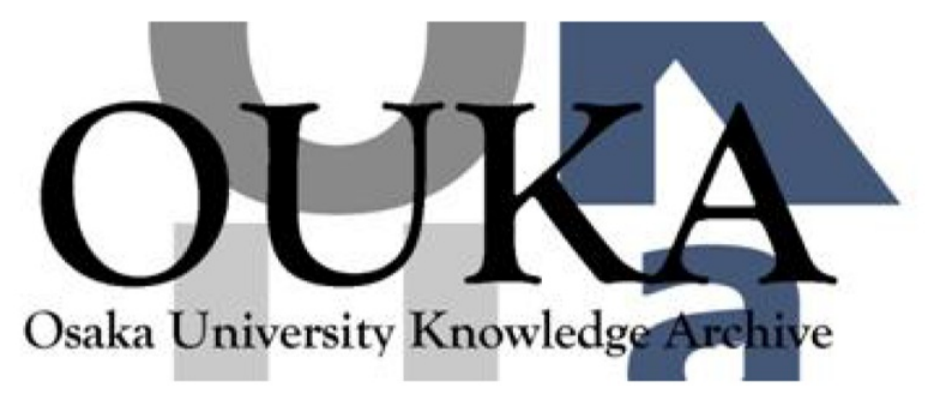

\begin{tabular}{|c|l|}
\hline Title & $\begin{array}{l}\text { Spin Pumping from Rashba Spin-Orbit-Coupled } \\
\text { Electron Systems Driven by Electric Dipole Spin } \\
\text { Resonance }\end{array}$ \\
\hline Author(s) & Hattori, Kiminori \\
\hline Citation & $\begin{array}{l}\text { Journal of the Physical Society of Japan. 77(3) } \\
\text { p. } 034707\end{array}$ \\
\hline Issue Date & $2008-03$ \\
\hline oaire:version & AM \\
\hline URL & https://hdl. handle. net/11094/3266 \\
\hline rights & \\
\hline Note & \\
\hline
\end{tabular}

Osaka University Knowledge Archive : OUKA

https://ir. Library. osaka-u. ac. jp/

Osaka University 


\title{
Spin Pumping from Rashba Spin-Orbit-Coupled Electron Systems Driven by Electric Dipole Spin Resonance
}

\author{
Kiminori HATTORI* \\ Department of Systems Innovation, Graduate School of Engineering Science, Osaka \\ University, Toyonaka, Osaka 560-8531
}

This paper analyzes spin currents pumped from Rashba spin-orbit coupled two-dimensional electron systems in electric dipole spin resonance (EDSR), on the basis of the nonequilibrium Green's function formalism. In the ballistic transport regime, the EDSR-induced spin pumping efficiently occurs for the finite-sized system smaller than the spin precession length. In the diffusive transport regime, the spin pumping is remarkably enhanced with increasing static disorder while the pumped spin dephases to a certain degree due to the D'yakonov-Perel' mechanism. The spin dephasing is controlled by reducing the system size compared with the precession length irrespective of the degree of disorder.

KEYWORDS: spin pumping, spin-orbit coupling, electric dipole spin resonance, two-dimensional system, spin transport, spin relaxation

*E-mail: hattori@ee.es.osaka-u.ac.jp 


\section{Introduction}

Spintronics, which aims to manipulate electron spin in addition to charge in solid-state systems, has grown to become a very active field in condensed matter physics. One central issue in this field is how to efficiently generate spin currents in nonmagnetic metals or semiconductors. Many methodologies have been proposed to date. Several approaches are based on purely magnetic means, including spin pumping from paramagnetic quantum dots or finite-sized conductors in paramagnetic resonance, ${ }^{1,2)}$ as well as a spin battery consisting of a ferromagnet in ferromagnetic resonance attached to a nonmagnetic conductor. ${ }^{3)}$ The ubiquitous presence of spin-orbit (SO) coupling in conventional semiconductors and quantum heterostructures has also been considered as a tool to manipulate spin current. The spin Hall effects in the SO coupled systems have been extensively investigated, providing an effective way to produce transverse spin flux in response to a longitudinal electric field or charge current. $^{4,5)}$ The parametric quantum pumping by cyclic variations of system parameters in the presence of the SO coupling has attracted considerable interest for spin current generation. ${ }^{6-8)}$ A few spin pumping mechanisms have been proposed that utilize a time-dependent gate potential that modulates the Rashba SO coupling in an asymmetric quantum well. ${ }^{9,10)}$

It is known that a versatile and efficient spin control is enabled in the presence of SO coupling by electric dipole spin resonance (EDSR), ${ }^{11-19)}$ where a time-dependent external electric field gives rise to a time-dependent internal magnetic field and couples to spin degrees of freedom. An arbitrary spin rotation is feasible by an adequate 
configuration of the time-dependent electric field and a static magnetic field defining a quantization axis for the spin. This is analogous to standard paramagnetic resonance techniques. This paper proposes a new spin pumping scheme exploiting EDSR, and pursues a detailed theoretical analysis of spin currents pumped from Rashba SO coupled two-dimensional (2D) electron systems, on the basis of the nonequilibrium Green's function (NEGF) formalism. ${ }^{20-23)}$ Considering a specific geometry with the external electric and magnetic fields that are parallel and in-plane, we show that spin currents are efficiently generated by the EDSR mechanism in both ballistic and diffusive transport regimes.

\section{Theoretical Analysis and Formulation}

In this paper we shall work in units where $\hbar=e=1$. A general form of the Hamiltonian describing the Rashba SO coupled 2D electron gas in the $x y$ plane subjected to external electromagnetic fields is expressed as

$$
H=\frac{1}{2 m}(\mathbf{k}+\mathbf{A})^{2}+\lambda\left[(\mathbf{k}+\mathbf{A}) \times \mathbf{e}_{z}\right] \cdot \mathbf{S}+\gamma \mathbf{B} \cdot \mathbf{S},
$$

where $\mathbf{k}$ is the canonical momentum operator, $\mathbf{S}$ is the spin operator, $m$ is the electron mass, $\lambda$ is the SO coupling strength, $\gamma$ is the gyromagnetic ratio, and $\mathbf{e}_{\mu}$ ( $\mu=x, y, z)$ is the unit vector in Cartesian coordinates. As shown in Fig. 1, we consider particularly the static magnetic field $\mathbf{B}=\mathbf{e}_{y} \bar{B}$ and the oscillating electric field $\mathbf{E}(t)=\mathbf{e}_{y} \tilde{E} \sin \omega t$, which are parallel and in-plane and amount to the associated in-plane 
vector potential $\mathbf{A}(t)=\mathbf{e}_{y}(\tilde{E} / \omega) \cos \omega t$. Then the total Hamiltonian $H(t)=\bar{H}+V(t)$, with $A^{2}$ omitted for simplicity, consists of the static part $\bar{H}=H_{0}+H_{\mathrm{so}}+H_{\mathrm{Z}}$ and the time-dependent part $V(t)=V_{0}(t)+V_{\mathrm{SO}}(t)$, including $H_{0}=k^{2} / 2 m, \quad H_{\mathrm{SO}}=\gamma \mathbf{B}_{\mathrm{SO}} \cdot \mathbf{S}$, $H_{\mathrm{Z}}=\gamma \mathbf{B} \cdot \mathbf{S}, \quad V_{0}(t)=\mathbf{A}(t) \cdot \mathbf{k} / m$, and $V_{\mathrm{SO}}(t)=\tilde{\gamma} \tilde{\mathbf{B}}(t) \cdot \mathbf{S}$, where the internal magnetic fields induced by the SO coupling are defined by $\gamma_{\mathrm{sO}}=\lambda \mathbf{k} \times \mathbf{e}_{z}$ and $\tilde{\mathbf{B}}(t)=\lambda \mathbf{A}(t) \times \mathbf{e}_{z}$. The oscillating internal field $\tilde{\mathbf{B}}(t)$ that is in-plane and perpendicular to the static external field $\mathbf{B}$ constitutes a standard EDSR setup. ${ }^{15)}$

The Schrödinger equation $i \hbar \partial \psi / \partial t=H \psi$ in terms of the fermion field operator $\psi$ leads to the usual sourceless continuity equation

$$
\frac{\partial}{\partial} \rho(\mathbf{r}, t)+\nabla \cdot \mathbf{j}(\mathbf{r}, t)=0
$$

for the charge density operator $\rho=\psi^{\dagger} \psi$ and the charge current density operator $\mathbf{j}=\operatorname{Re} \psi^{\dagger} \mathbf{v} \psi+\rho \mathbf{A} / m$. In the latter equation, $\mathbf{v}=\mathbf{v}_{0}+\mathbf{v}_{\text {So }}$ is the kinetic velocity operator consisting of the canonical velocity $\mathbf{v}_{0}=\mathbf{k} / m$ and the spin-dependent anomalous velocity due to the SO coupling $\mathbf{v}_{\mathrm{SO}}=\lambda \mathbf{e}_{z} \times \mathbf{S}$, and $\operatorname{Re} \mathcal{A}$ is defined as $\operatorname{Re} \mathcal{A}=\left(\mathcal{A}+\mathcal{A}^{\dagger}\right) / 2$. Note that in a second-quantized form, the time-dependent potentials are expressed as $V_{0}(t)=\int d \mathbf{r} \mathbf{j}_{0}(\mathbf{r}, t) \cdot \mathbf{A}(t)$ and $V_{\mathrm{SO}}(t)=\int d \mathbf{r} \mathbf{j}_{\mathrm{SO}}(\mathbf{r}, t) \cdot \mathbf{A}(t)$, where $\mathbf{j}_{0}=\operatorname{Re} \psi^{\dagger} \mathbf{v}_{0} \psi$ and $\mathbf{j}_{\mathrm{sO}}=\psi^{\dagger} \mathbf{v}_{\mathrm{SO}} \psi$. The spin density operator $\rho_{\mu}=\psi^{\dagger} S_{\mu} \psi$ and the spin current density operator $\mathbf{j}_{\mu}=\operatorname{Re} \psi^{\dagger} S_{\mu} \mathbf{v} \psi+\rho_{\mu} \mathbf{A} / m$ obey

$$
\frac{\partial}{\partial t} \rho_{\mu}(\mathbf{r}, t)+\nabla \cdot \mathbf{j}_{\mu}(\mathbf{r}, t)=g_{\mu}(\mathbf{r}, t)
$$


where the operator $g_{\mu}=\mathbf{e}_{\mu} \cdot \operatorname{Re} \psi^{\dagger} \gamma\left(\mathbf{B}_{\mathrm{SO}}+\mathbf{B}+\tilde{\mathbf{B}}\right) \times \mathbf{S} \psi$ represents the spin- $S_{\mu}$ component of spin torque density. Each component is explicitly expressed as

$$
\begin{aligned}
& g_{x}=-\lambda \operatorname{Re} \psi^{\dagger} S_{z} k_{x} \psi+\bar{\omega} \rho_{z}, \\
& g_{y}=-\lambda \operatorname{Re} \psi^{\dagger} S_{z} k_{y} \psi-2 \tilde{\omega} \rho_{z} \cos \omega t, \\
& g_{z}=\lambda \operatorname{Re} \psi^{\dagger} \mathbf{S} \cdot \mathbf{k} \psi-\bar{\omega} \rho_{x}+2 \tilde{\omega} \rho_{y} \cos \omega t,
\end{aligned}
$$

where $\bar{\omega}=\gamma \bar{B}$ and $\tilde{\omega}=\lambda \tilde{E} / 2 \omega$. In view of the spin-current continuity equation, the spin pumping can be interpreted as being due to the spin torque that causes spin nonconservation. ${ }^{2)}$

First of all, we deal with the spatially-integrated spin torque $G_{\mu}(t)=\int d \mathbf{r} g_{\mu}(\mathbf{r}, t)$ generated in an infinitely-large homogeneous system. Because of the translation invariance, the Hamiltonian $H(t)=\bar{H}+V(t)$ is diagonal in the momentum basis. The static part $\bar{H}=H_{0}+H_{\mathrm{sO}}+H_{\mathrm{z}}$ forms the spin-split subbands with the energy $\varepsilon_{\mathbf{k}, s}=\varepsilon_{\mathbf{k}}+s \omega_{\mathbf{k}} / 2 \quad(s= \pm 1)$, where $\varepsilon_{\mathbf{k}}=k^{2} / 2 m$ denotes the free-particle dispersion and $\omega_{\mathbf{k}}=\sqrt{\left(\lambda k_{y}\right)^{2}+\left(\bar{\omega}-\lambda k_{x}\right)^{2}}$ is the Zeeman energy splitting due to the total static field $\mathbf{B}_{\mathrm{SO}}+\mathbf{B}$. Treating the time-dependent part $V(t)=V_{0}(t)+V_{\mathrm{SO}}(t)$ as a perturbation, the Kubo formula ${ }^{22)}$ is used to evaluate the spin torque $\left\langle G_{\mu}(t)\right\rangle$. It is obvious that there is no contribution due to $V_{0}(t)$ since it is spin-independent. The dc component of spin torque, $G_{\mu}=\lim _{T \rightarrow \infty} T^{-1} \int_{-T / 2}^{T / 2} d t\left\langle G_{\mu}(t)\right\rangle$, arises from the coupling between oscillations involved in the operators $G_{\mu}(t)$ and $V_{\mathrm{So}}(t)$, and is found to be 


$$
G_{y}=\frac{\pi}{2} \tilde{\omega}^{2} \sum_{\mathbf{k}}\left[f\left(\varepsilon_{\mathbf{k}}-\frac{\omega_{\mathbf{k}}}{2}\right)-f\left(\varepsilon_{\mathbf{k}}+\frac{\omega_{\mathbf{k}}}{2}\right)\right] \sin \phi_{\mathbf{k}}\left[\delta\left(\omega-\omega_{\mathbf{k}}\right)+\delta\left(\omega+\omega_{\mathbf{k}}\right)\right],
$$

and $G_{x}=G_{z}=0$, where $f(\varepsilon)$ is the Fermi-Dirac distribution function and $\tan \phi_{\mathbf{k}}=\left(\bar{\omega}-\lambda k_{x}\right) / \lambda k_{y}$. The delta function $\delta\left(\omega \pm \omega_{\mathbf{k}}\right)$ explains the spin resonance produced by the electron transition between the spin-split subbands $\varepsilon_{\mathbf{k} \pm}=\varepsilon_{\mathbf{k}} \pm \omega_{\mathbf{k}} / 2$. The resonance occurs only when the condition $\omega= \pm \omega_{\mathbf{k}}$ is met, which tends to restrict momentum states contributing to the spin torque $G_{y}$. It is easily found that neglecting the SO coupling term $H_{\mathrm{SO}}$ in $\bar{H}$ results in an approximation to $G_{y}$ in lowest nonvanishing order in $\lambda$. In such a case, $G_{y}$ becomes

$$
G_{y}=\frac{\pi}{2} \tilde{\omega}^{2}[\delta(\omega-\bar{\omega})+\delta(\omega+\bar{\omega})] \sum_{\mathbf{k}}\left[f\left(\varepsilon_{\mathbf{k}}-\frac{\bar{\omega}}{2}\right)-f\left(\varepsilon_{\mathbf{k}}+\frac{\bar{\omega}}{2}\right)\right],
$$

which is divergent at $\omega= \pm \bar{\omega}$. It can be seen from comparing these two expressions that the spin torque $G_{y}$ is significantly diminished by $H_{\text {so }}$ contained in $\bar{H}$. The implications derived for infinitely large systems will be reexamined quantitatively when discussing the numerical results obtained for finite-sized systems.

The oscillating field $\tilde{\mathbf{B}}(t)=\mathbf{e}_{x}(2 \tilde{\omega} / \gamma) \cos \omega t$ can be decomposed into two components $\tilde{\mathbf{B}}^{( \pm)}(t)=(\tilde{\omega} / \gamma)\left(\mathbf{e}_{x} \cos \omega t \mp \mathbf{e}_{z} \sin \omega t\right)$ rotating in the $x z$ plane. The counterclockwise component $\tilde{\mathbf{B}}^{(+)}(t)$ and the clockwise component $\tilde{\mathbf{B}}^{(-)}(t)$ (seen from the positive $y$-side) are interchanged by simply reversing the sign of $\omega$. For mathematical convenience, the analysis of the spin resonance customarily employs 
$\tilde{\mathbf{B}}^{( \pm)}(t)$ instead of $\tilde{\mathbf{B}}(t){ }^{24)}$ We here briefly summarize the analytical results derived for the rotating fields $\tilde{\mathbf{B}}^{( \pm)}(t)$. Defining the corresponding operators $V_{\mathrm{SO}}^{( \pm)}(t)$ and $G_{\mu}^{( \pm)}(t)$ by simply replacing $\tilde{\mathbf{B}}(t)$ with $\tilde{\mathbf{B}}^{( \pm)}(t)$, the time-averaged spin torques $G_{\mu}^{( \pm)}$ for the rotating fields $\tilde{\mathbf{B}}^{( \pm)}(t)$ are calculated to be

$$
G_{y}^{( \pm)}=\frac{\pi}{8} \tilde{\omega}^{2} \sum_{\mathbf{k}, s}\left[f\left(\varepsilon_{\mathbf{k},-s}\right)-f\left(\varepsilon_{\mathbf{k}, s}\right)\right]\left(\sin \phi_{\mathbf{k}}+s\right)^{2} \delta\left(\omega \mp s \omega_{\mathbf{k}}\right)
$$

and again $G_{x}^{( \pm)}=G_{z}^{( \pm)}=0$. Equations (5) and (7) satisfy the simple decomposition $G_{y}=G_{y}^{(+)}+G_{y}^{(-)}$for $\tilde{\mathbf{B}}(t)=\tilde{\mathbf{B}}^{(+)}(t)+\tilde{\mathbf{B}}^{(-)}(t)$. Notice that this property is not a direct consequence from the perturbation theory because the time-dependent potentials $V_{\mathrm{SO}}^{( \pm)}(t)$ and the spin torque operators $G_{\mu}^{( \pm)}(t)$ are simultaneously adapted to $\tilde{\mathbf{B}}^{( \pm)}(t)$.

We next turn to the theoretical analysis for finite-sized systems. A finite-sized spin pumping system is modeled by considering a sample with the SO coupling in contact with two semi-infinite ideal leads maintained at the same electrochemical potential. In practice, the electric field can be generated by a pair of gates as sketched in Fig. 1, between which an ac voltage of frequency $\omega$ is applied from an external circuit (not shown). In the leads that transport spin, the SO coupling is removed in order to probe the spin current. In the tight-binding representation on a square lattice with lattice spacing $a$, the Hamiltonian describing the sample region reads

$$
\begin{aligned}
& H_{0}=-t_{0} \sum_{\mathbf{r}, \sigma} \sum_{ \pm}\left(c_{\mathbf{r} \sigma}^{\dagger} c_{\mathbf{r} \pm \mathbf{a} \sigma}+c_{\mathbf{r} \sigma}^{\dagger} c_{\mathbf{r} \pm \mathbf{b} \sigma}\right)+4 t_{0} \sum_{\mathbf{r}, \sigma} c_{\mathbf{r} \sigma}^{\dagger} c_{\mathbf{r} \sigma}, \\
& H_{\mathrm{SO}}=\frac{\lambda}{4 a} \sum_{\mathbf{r}, \sigma} \sum_{ \pm}\left( \pm \sigma c_{\mathbf{r} \sigma}^{\dagger} c_{\mathbf{r} \pm \mathbf{a},-\sigma} \mp i c_{\mathbf{r} \sigma}^{\dagger} c_{\mathbf{r} \pm \mathbf{b},-\sigma}\right),
\end{aligned}
$$




$$
\begin{aligned}
& H_{\mathrm{Z}}=\frac{\bar{\omega}}{2 i} \sum_{\mathbf{r}, \sigma} \sigma c_{\mathbf{r} \sigma}^{\dagger} c_{\mathbf{r},-\sigma}, \\
& V_{\mathrm{SO}}^{( \pm)}(t)=\frac{\tilde{\omega}}{2} \sum_{\mathbf{r}, \sigma}\left(c_{\mathbf{r} \sigma}^{\dagger} c_{\mathbf{r},-\sigma} \cos \omega t \mp \sigma c_{\mathbf{r} \sigma}^{\dagger} c_{\mathbf{r} \sigma} \sin \omega t\right),
\end{aligned}
$$

where $c_{\mathbf{r} \sigma}\left(c_{\mathbf{r} \sigma}^{\dagger}\right)$ is the annihilation (creation) operator of an electron at position $\mathbf{r}$ with spin $\sigma(= \pm 1$ corresponding to spin- $\uparrow$ and $\downarrow$ states for the quantization axis along $z$ ), $\mathbf{a}=a \mathbf{e}_{x}$ and $\mathbf{b}=a \mathbf{e}_{y}$ are the unit lattice vectors, and $t_{0}=1 / 2 m a^{2}$ is the hopping energy. The Larmor frequency $\tilde{\omega}$ for the internal magnetic field is expressed as $\tilde{\omega}=\eta \lambda / 2 a$ with $\eta=\tilde{E} a / \omega$. In what follows, we consider the spin current produced by a general harmonic potential $V(t)=V \exp (-i \omega t)+V^{\dagger} \exp (i \omega t)$, on the basis of the NEGF formalism. Note that the pumping potential $V(t)$ is spin-dependent when considering EDSR.

Two nonequilibrium one-particle propagators, the retarded Green's function $G^{+}\left(t, t^{\prime}\right)$ and the lesser Green' function $G^{<}\left(t, t^{\prime}\right)$, are defined by

$$
\begin{aligned}
& {\left[G^{+}\left(t, t^{\prime}\right)\right]_{\mathbf{r} \sigma ; \mathbf{r}^{\prime} \sigma^{\prime}}=-i\left\langle\left\{c_{\mathbf{r} \sigma}(t), c_{\mathbf{r}^{\prime} \sigma^{\prime}}^{\dagger}\left(t^{\prime}\right)\right\}\right\rangle \theta\left(t-t^{\prime}\right),} \\
& {\left[G^{<}\left(t, t^{\prime}\right)\right]_{\mathbf{r} \sigma ; \mathbf{r}^{\prime} \sigma^{\prime}}=i\left\langle c_{\mathbf{r}^{\prime} \sigma^{\prime}}^{\dagger}\left(t^{\prime}\right) c_{\mathbf{r} \sigma}(t)\right\rangle .}
\end{aligned}
$$

The retarded Green's function obeys the following equation of motion:

$$
i \frac{\partial}{\partial} G^{+}\left(t, t^{\prime}\right)-H(t) G^{+}\left(t, t^{\prime}\right)-\int_{-\infty}^{\infty} d t^{\prime \prime} \Sigma^{+}\left(t-t^{\prime \prime}\right) G^{+}\left(t^{\prime \prime}, t^{\prime}\right)=\delta\left(t-t^{\prime}\right)
$$

where $\Sigma^{+}(t)=\Sigma_{\alpha} \Sigma_{\alpha}^{+}(t)$ with $\Sigma_{\alpha}^{+}(t)$ being the retarded self-energy due to lead $\alpha$ 
connected to the sample. After applying the double-time Fourier transformation, the equation of motion becomes

$$
G^{+}\left(\varepsilon, \varepsilon^{\prime}\right)=2 \pi \delta\left(\varepsilon-\varepsilon^{\prime}\right) g^{+}\left(\varepsilon^{\prime}\right)+G^{+}\left(\varepsilon, \varepsilon^{\prime}+\omega\right) V g^{+}\left(\varepsilon^{\prime}\right)+G^{+}\left(\varepsilon, \varepsilon^{\prime}-\omega\right) V^{\dagger} g^{+}\left(\varepsilon^{\prime}\right)
$$

where $g^{+}(\varepsilon)=\left[\varepsilon-\bar{H}-\Sigma^{+}(\varepsilon)\right]^{-1}$ is the Fourier transform of the retarded Green's function $g^{+}\left(t-t^{\prime}\right)$ that corresponds to $G^{+}\left(t, t^{\prime}\right)$ in the absence of the pumping potential $V(t)$ and hence depends only on the time difference $t-t^{\prime}$. The solution of eq. (12) is restricted to the form $G_{n}^{+}(\varepsilon)=G^{+}(\varepsilon, \varepsilon+n \omega)$, which contains a couple of frequencies shifted by $n \omega$ (where $n$ is an integer), reflecting multi-quantum contributions from $V(t)$. Therefore, the equation of motion is reduced to

$$
G_{n}^{+}(\varepsilon)=2 \pi \delta(n \omega) g_{n}^{+}(\varepsilon)+G_{n+1}^{+}(\varepsilon) V g_{n}^{+}(\varepsilon)+G_{n-1}^{+}(\varepsilon) V^{\dagger} g_{n}^{+}(\varepsilon)
$$

with $g_{n}^{+}(\varepsilon)=g^{+}(\varepsilon+n \omega)$. Equation (13) can be solved in an iterative manner ${ }^{8,25)}$ by $\hat{G}_{0}^{+}=\left[\left(g_{0}^{+}\right)^{-1}-\Sigma_{0}^{+}\right]^{-1}$ for $n=0, \hat{G}_{n}^{+}=\hat{G}_{n-1}^{+} V^{\dagger} g_{n}^{+} \alpha_{n}$ for $n \geq 1$, and $\hat{G}_{n}^{+}=\hat{G}_{n+1}^{+} V g_{n}^{+} \beta_{n}$ for $n \leq-1$. Here, $G_{n}^{+}=2 \pi \delta(0) \hat{G}_{n}^{+}, \quad \Sigma_{0}^{+}=V^{\dagger} g_{1}^{+} \alpha_{1} V+V g_{-1}^{+} \beta_{-1} V^{\dagger}$, and the dimensionless coefficients $\alpha_{n}$ and $\beta_{n}$ follow the relations $\alpha_{n}\left(1-V^{\dagger} g_{n+1}^{+} \alpha_{n+1} V g_{n}^{+}\right)=1$ and $\beta_{n}\left(1-V g_{n-1}^{+} \beta_{n-1} V^{\dagger} g_{n}^{+}\right)=1$, which form solutions expressed as continued fractions.

The spin- $S_{\mu}$ component of spin current flowing into lead $\alpha$ is defined by $J_{\alpha}^{\mu}(t)=\left\langle d S_{\alpha}^{\mu}(t) / d t\right\rangle$ with the spin operator $S_{\alpha}^{\mu}=\sum_{\mathbf{r} \in \alpha} \sum_{\sigma, \sigma^{\prime}} C_{\mathbf{r} \sigma}^{\dagger}\left[S_{\mu}\right]_{\sigma \sigma^{\prime}} C_{\mathbf{r} \sigma^{\prime}}$ for electrons in lead $\alpha$. We postulate the conservation of spin $S_{\mu}$ in the isolated lead $\alpha$ (otherwise, the pumped spin current $J_{\alpha}^{\mu}$ can not be probed). Then, the Heisenberg 
equation for $S_{\alpha}^{\mu}$ leads to

$$
J_{\alpha}^{\mu}(t)=-2 \operatorname{Re} \int_{-\infty}^{\infty} d t^{\prime} \operatorname{Tr}\left\{S_{\mu}\left[G^{+}\left(t, t^{\prime}\right) \Sigma_{\alpha}^{<}\left(t^{\prime}-t\right)+G^{<}\left(t, t^{\prime}\right) \Sigma_{\alpha}^{-}\left(t^{\prime}-t\right)\right]\right\},
$$

where $\Sigma_{\alpha}^{-}(t)=\left[\Sigma_{\alpha}^{+}(-t)\right]^{\dagger}$ and $\Sigma_{\alpha}^{<}(t)$ is the lesser self-energy due to lead $\alpha$. The lesser self-energy is expressed as $\Sigma_{\alpha}^{<}(\varepsilon)=i f(\varepsilon) \Gamma_{\alpha}(\varepsilon)$ in the Fourier space, where $\Gamma_{\alpha}(\varepsilon)=-2 \operatorname{Im} \Sigma_{\alpha}^{+}(\varepsilon)$ and $\operatorname{Im} \mathcal{A}=\left(\mathcal{A}-\mathcal{A}^{\dagger}\right) / 2 i$. The lesser Green's function satisfies the Keldysh equation

$$
G^{<}\left(t, t^{\prime}\right)=\int_{-\infty}^{\infty} d t_{1} \int_{-\infty}^{\infty} d t_{2} G^{+}\left(t, t_{1}\right) \Sigma^{<}\left(t_{1}-t_{2}\right) G^{-}\left(t_{2}, t^{\prime}\right)
$$

with $\Sigma^{<}(t)=\Sigma_{\alpha} \Sigma_{\alpha}^{<}(t)$, and is related to the retarded Green's function $G^{+}\left(t, t^{\prime}\right)=\left[G^{-}\left(t^{\prime}, t\right)\right]^{\dagger}$. The time-averaged spin current, $J_{\alpha}^{\mu}=\lim _{T \rightarrow \infty} T^{-1} \int_{-T / 2}^{T / 2} d t J_{\alpha}^{\mu}(t)$, is eventually formulated as

$$
J_{\alpha}^{\mu}=\frac{1}{2 \pi} \sum_{n=-\infty}^{\infty} \int_{-\infty}^{\infty} d \varepsilon \operatorname{Tr}\left[S_{\mu} \Gamma_{\alpha}(\varepsilon) \hat{G}_{n}^{+}(\varepsilon) \Gamma_{n}(\varepsilon) \hat{G}_{n}^{-}(\varepsilon)\right]\left[f_{n}(\varepsilon)-f(\varepsilon)\right]
$$

where $\hat{G}_{n}^{-}(\varepsilon)=\left[\hat{G}_{n}^{+}(\varepsilon)\right]^{\dagger}, \quad \Gamma_{n}(\varepsilon)=-2 \operatorname{Im} \Sigma^{+}(\varepsilon+n \omega), \quad$ and $\quad f_{n}(\varepsilon)=f(\varepsilon+n \omega) . \quad$ An extension to more general cases when the electrochemical potential is different for each lead is straightforward, for which we obtain

$$
J_{\alpha}^{\mu}=\frac{1}{2 \pi} \sum_{n=-\infty}^{\infty} \sum_{\beta} \int_{-\infty}^{\infty} d \varepsilon \operatorname{Tr}\left[S_{\mu} \Gamma_{\alpha}(\varepsilon) \hat{G}_{n}^{+}(\varepsilon) \Gamma_{\beta n}(\varepsilon) \hat{G}_{n}^{-}(\varepsilon)\right]\left[f_{\beta n}(\varepsilon)-f_{\alpha}(\varepsilon)\right],
$$


where $\Gamma_{\alpha n}(\varepsilon)=\Gamma_{\alpha}(\varepsilon+n \omega), \quad f_{\alpha n}(\varepsilon)=f_{\alpha}(\varepsilon+n \omega)$, and $f_{\alpha}(\varepsilon)$ is the Fermi function in lead $\alpha$. It is easily shown that eqs. (16) and (17) reproduce the previous results obtained for the parametric quantum spin pumping in the presence of the SO coupling, as well as for the spin pumping due to paramagnetic resonance in the absence of the SO coupling. ${ }^{1,2)}$

There are two limiting cases where reduced expressions are derived from eq. (16). In the adiabatic regime $(\omega \rightarrow 0)$, the pumped spin per one pumping cycle, $P_{\alpha}^{\mu}=(2 \pi / \omega) J_{\alpha}^{\mu}$, can be written as

$$
P_{\alpha}^{\mu}=-\sum_{n=-\infty}^{\infty} n \operatorname{Tr}\left[S_{\mu} \Gamma_{\alpha}(E) \hat{G}_{n}^{+}(E) \Gamma_{n}(E) \hat{G}_{n}^{-}(E)\right]
$$

at zero temperature, indicating that the spin pumping takes place via current-carrying states at the Fermi level $E$. In the perturbative regime ( $V \rightarrow 0)$, one obtains

$$
\begin{aligned}
J_{\alpha}^{\mu} & =\frac{1}{2 \pi} \int_{-\infty}^{\infty} d \varepsilon \operatorname{Tr}\left[S_{\mu} \Gamma_{\alpha}(\varepsilon) g^{+}(\varepsilon) V^{\dagger} A_{1}(\varepsilon) V g^{-}(\varepsilon)\right]\left[f_{1}(\varepsilon)-f(\varepsilon)\right] \\
& +\frac{1}{2 \pi} \int_{-\infty}^{\infty} d \varepsilon \operatorname{Tr}\left[S_{\mu} \Gamma_{\alpha}(\varepsilon) g^{+}(\varepsilon) V A_{-1}(\varepsilon) V^{\dagger} g^{-}(\varepsilon)\right]\left[f_{-1}(\varepsilon)-f(\varepsilon)\right]
\end{aligned},
$$

where $A_{n}(\varepsilon)=g_{n}^{+}(\varepsilon) \Gamma_{n}(\varepsilon) g_{n}^{-}(\varepsilon)$ is the spectral function. This expression clearly shows a quadratic dependence on the magnitude of the pumping potential. It is also demonstrated from eq. (19) through the unitary transformation: $S_{y} \rightarrow S_{z}$ and $S_{z} \rightarrow-S_{y}$ that $J_{\alpha}^{x}=J_{\alpha}^{z}=0$ and $J_{\alpha}^{y}=J_{\alpha}^{y(+)}+J_{\alpha}^{y(-)}$ when $\bar{H}=H_{0}+H_{\mathrm{Z}}$ and $V(t)=V_{\mathrm{SO}}^{(+)}(t)+V_{\mathrm{SO}}^{(-)}(t)$. These relations indicate that if $\lambda$ is sufficiently small, only the spin- $S_{y}$ current is 
pumped, and the spin current $J_{\alpha}^{y}$ generated by the oscillating field $\tilde{\mathbf{B}}(t)$ is decomposed into two contributions $J_{\alpha}^{y( \pm)}$ due to the rotating fields $\tilde{\mathbf{B}}^{( \pm)}(t)$. In this case, we find that the total outward flow of spin current, $J_{y}^{( \pm)}=\sum_{\alpha} J_{\alpha}^{y( \pm)}$, is expressed simply with the spin quantization axis along $y$ as

$$
J_{y}^{( \pm)}=\frac{\tilde{\omega}^{2}}{8 \pi} \int_{-\infty}^{\infty} d \varepsilon \operatorname{Tr}\left\{\left[A_{0}(\varepsilon)\right]_{\downarrow \downarrow}\left[A_{ \pm 1}(\varepsilon)\right]_{\uparrow \uparrow}\right\}\left[f(\varepsilon)-f_{ \pm 1}(\varepsilon)\right]
$$

where the trace is taken over orbital degrees of freedom.

Before ending this section, we compare the perturbative result for spin current based on the NEGF formalism with that for spin torque derived from the Kubo formula for an infinitely-large homogeneous system. The perturbation analysis for the spin torque can be generalized for an arbitrary pumping potential $V$ by introducing the spin torque operator $G_{\mu}=-i\left[S_{\mu}, H\right]$ in terms of the Heisenberg equation. Then, the Kubo formula yields the time-averaged spin torque represented as

$$
\begin{aligned}
G_{\mu} & =2 \pi \int_{-\infty}^{\infty} d \varepsilon \operatorname{Tr}\left[S_{\mu} V^{\dagger} \delta(\varepsilon-\bar{H}+\omega) V \delta(\varepsilon-\bar{H})\right]\left[f_{1}(\varepsilon)-f(\varepsilon)\right] \\
& +2 \pi \int_{-\infty}^{\infty} d \varepsilon \operatorname{Tr}\left[S_{\mu} \delta(\varepsilon-\bar{H}) V \delta(\varepsilon-\bar{H}-\omega) V^{\dagger}\right]\left[f_{-1}(\varepsilon)-f(\varepsilon)\right]
\end{aligned} .
$$

Comparing eq. (21) with eq. (19) leads to the equality $J_{\mu}=\sum_{\alpha} J_{\alpha}^{\mu}=G_{\mu}$ for an infinite system when $\left[S_{\mu}, \bar{H}\right]=0 .{ }^{2)}$ Clearly, the simple identity $J_{\mu}=G_{\mu}$ no longer holds for infinitely-large Rashba SO coupled systems with $\lambda \neq 0$, since $H_{\text {so }}$ does not commute with $S_{\mu}$. 


\section{Numerical Calculation and Discussion}

Numerical calculations based on the NEGF formalism have been performed for a quantitative study of the EDSR-induced spin pumping from Rashba SO coupled electron systems. The model system consists of a central square sample of size $L \times L$ with SO coupling, to which two semi-infinite leads (labeled by $\alpha=1,2$ ) of width $L$ are attached, as shown in Fig. 1. The two leads are of the same electrochemical potential. We neglect the spin-independent potential $V_{0}(t)$, which does not contribute to the time-averaged spin torque. ${ }^{26)}$ The Zeeman interaction $H_{\mathrm{z}}$ due to the static field $\mathbf{B}$ and the time-dependent pumping potential $V(t)=V_{\mathrm{SO}}^{(+)}(t)$ due to the rotating field $\tilde{\mathbf{B}}^{(+)}(t)$ are assumed to exist only in the sample region. The pumping potential $V_{\mathrm{SO}}^{(+)}(t)$ can be employed without loss of generality, since the bulk of the numerical analysis in this study addresses a small $\lambda$ case where $J_{\alpha}^{\mu} \cong J_{\alpha}^{\mu(+)}+J_{\alpha}^{\mu(-)}$ for $\tilde{\mathbf{B}}(t)=\tilde{\mathbf{B}}^{(+)}(t)+\tilde{\mathbf{B}}^{(-)}(t)$. For conciseness, the notation $(+)$ is omitted for the spin current $J_{\alpha}^{\mu}$ and the pumped spin $P_{\alpha}^{\mu}$ for the remainder of this paper. The calculation assumes zero temperature and the perturbative regime where $J_{\alpha}^{\mu}$ is a quadratic function of $\eta=\tilde{E} a / \omega$. The numerical results shown below are presented in the normalized form $\hat{J}_{\alpha}^{\mu}=J_{\alpha}^{\mu} / \eta^{2}$ and $\hat{P}_{\alpha}^{\mu}=P_{\alpha}^{\mu} / \eta^{2}$. In the following, the hopping energy is taken as the energy unit $\left(t_{0}=1\right)$, and the lattice spacing as the length unit $(a=1)$. The parameters are normally $E=0.1, \bar{\omega}=0$, and $\omega \rightarrow 0$ (which corresponds to the adiabatic regime), unless otherwise stated.

Before discussing the numerical results, it may be appropriate to describe some general results proven from the symmetry of the two-terminal model assumed for the 
calculation. Consider the unitary transformation: $y \rightarrow-y, z \rightarrow-z, S_{x} \rightarrow-S_{x}$, and $S_{z} \rightarrow-S_{z}$, and the simultaneous sign change of the electric field: $\tilde{E} \rightarrow-\tilde{E}$. The total Hamiltonian including the pumping potential is invariant under this transformation. Since the pumped spin current $J_{\alpha}^{\mu}$ is an even function of $\tilde{E}$, the transformation simply reverses $J_{\alpha}^{x, z}$, leading to the relation that $J_{\alpha}^{x}=-J_{\alpha}^{x}$ and $J_{\alpha}^{z}=-J_{\alpha}^{z}$. Obviously, this means that

$$
\begin{aligned}
& J_{\alpha}^{x}=J_{\alpha}^{z}=0, \\
& P_{\alpha}^{x}=P_{\alpha}^{z}=0 .
\end{aligned}
$$

The absence of spin- $S_{x, z}$ currents accidentally coincides with the approximation derived in lowest nonvanishing order in $\lambda$ without assuming a specific model. Following the consideration given above, this property arises only from the symmetry of the system and holds irrespective of the SO coupling strength. Consider the unitary transformation: $x \rightarrow-x, \quad z \rightarrow-z, S_{y} \rightarrow-S_{y}$, and $S_{z} \rightarrow-S_{z}$, and the simultaneous sign changes of the static magnetic field and the pumping frequency: $\bar{\omega} \rightarrow-\bar{\omega}$ and $\omega \rightarrow-\omega$. The total Hamiltonian including the pumping potential is invariant under this transformation. The transformation interchanges the leads 1 and 2 and reverses the spin- $S_{y}$ current, yielding

$$
\begin{aligned}
& J_{1}^{y}(\omega, \bar{\omega})=-J_{2}^{y}(-\omega,-\bar{\omega}), \\
& P_{1}^{y}(\omega, \bar{\omega})=P_{2}^{y}(-\omega,-\bar{\omega}) .
\end{aligned}
$$


These analytical results equally apply to $\hat{J}_{\alpha}^{\mu}$ and $\hat{P}_{\alpha}^{\mu}$, and help to understand the numerical results described below.

Figure 2 displays the pumped spin $\hat{P}_{\alpha}^{y}$ calculated as a function of $\bar{\omega}$ in the adiabatic limit $\omega \rightarrow 0$ for various sample sizes $L$, assuming $\lambda / 4=10^{-4}$. The absence of spin- $S_{x, z}$ currents indicated by eq. (22) is confirmed by numerical calculation (not shown). The curves for $\hat{P}_{1}^{y}$ and $\hat{P}_{2}^{y}$ are nearly the same and exhibit a resonance peak around $\bar{\omega}=0$. A higher peak and a narrower linewidth are observed for larger samples. Figure 3 illustrates the sample-size dependencies of $\hat{P}_{1,2}^{y}$ at resonance. An oscillation observed in the numerical results is due to the subband formation in the finite-sized systems subjected to the lateral confinement. The sharp peak occurs when the bottom of the $1 \mathrm{D}$ subband (at which the density of states is singular) coincides with the Fermi energy $E=0.1$. Except for the oscillation, the overall feature suggests that the pumped spin basically follows a power-law dependence on $L$. A semiclassical consideration may be useful for interpreting these observations. $^{2)}$ For a finite-sized sample connected to two leads into which an electron can escape, the retarded self-energy can be approximately treated as $\Sigma^{+} \cong-i / \tau_{F}$, where $\tau_{F}=L / 2 v_{F}$ represents the average time an electron with Fermi velocity $v_{F}$ remains in the sample. ${ }^{21)}$ Applying the semiclassical approximation to eq. (20) leads to an analytical expression

$$
J_{y} \cong \frac{\tilde{\omega}^{2}}{2} \frac{\omega \tau_{F}}{1+\left[(\omega-\bar{\omega}) \tau_{F}\right]^{2}} N(E)
$$

for $\omega \cong \bar{\omega}$ and $|\omega| / 2<<E$, where $N(\varepsilon)=\operatorname{Tr} \delta\left(\varepsilon-H_{0}\right)$ is the density of states per spin. 
This formula neglects the SO coupling term $H_{\text {so }}$ included in $\bar{H}$ and is validated for a sufficiently small $\lambda$. Equation (24) deals with the nonadiabatic regime, and evaluates the resonance linewidth at $2 / \tau_{F}$ for finite-sized systems, which quantitatively agrees with the numerical results shown in Fig. 2. Assuming a free-particle dispersion, eq. (24) predicts

$$
P_{y}=\frac{2 \pi}{\omega} J_{y}=\frac{m \tilde{\omega}^{2} L^{3}}{4 v_{F}},
$$

at resonance $\omega=\bar{\omega}$. In Fig. 3, the analytical result ( $\hat{P}_{y} / 2$ per lead) is shown by dashed line, which satisfactorily explains the sample-size dependence observed in the numerical calculation.

Figure 4 compares the $\bar{\omega}$-dependencies in the limit $\omega \rightarrow 0$ and the $\omega$-dependencies at $\bar{\omega}=0$ calculated for different SO coupling strengths $\lambda$. When $\lambda$ is sufficiently small, the resonance occurs at $\bar{\omega}=0$ for $\hat{P}_{\alpha}^{y}(0, \bar{\omega})$ and $\omega=0$ for $\hat{P}_{\alpha}^{y}(\omega, 0)$, and these spectra are well overlapped with each other, as expected from eq. (24). When $\lambda$ becomes large, they are separated appreciably. The curve $\hat{P}_{1}^{y}(0, \bar{\omega})$ shifts toward the negative $\bar{\omega}$-side, while the curve $\hat{P}_{1}^{y}(\omega, 0)$ shifts toward the positive $\omega$-side. The opposite behaviors are exhibited by $\hat{P}_{2}^{y}(0, \bar{\omega})$ and $\hat{P}_{2}^{y}(\omega, 0)$, respectively. These displacements are almost the same in magnitude, showing the property

$$
\hat{P}_{1}^{y}(v, 0)=\hat{P}_{1}^{y}(0,-v)=\hat{P}_{2}^{y}(-v, 0)=\hat{P}_{2}^{y}(0, v) .
$$

The peak frequency shifting with $\lambda$ may be explained in terms of the resonance 
condition derived from the Kubo formula. The energy separation between the spin-split subbands approximates to $\omega_{\mathbf{k}} \cong\left|\bar{\omega}-\lambda k_{x}\right|$ up to first order in $\lambda$. In such a case, eq. (7) indicates that EDSR is produced via the momentum state satisfying the condition that $\omega= \pm\left(\bar{\omega}-\lambda k_{x}\right)$ for $G_{y}^{( \pm)}$. Considering two representative points $\left(k_{x}, k_{y}\right)=\left( \pm k_{F}, 0\right)$ on the Fermi circle amounts to the resonance condition $\omega=\bar{\omega} \pm \lambda k_{F}$ for $G_{y}^{(+)}$, which becomes $\bar{\omega}=\mp \lambda k_{F}$ for $\omega \rightarrow 0$ and $\omega= \pm \lambda k_{F}$ for $\bar{\omega}=0$. This argument is applicable particularly to a quasi-1D system, and reasonably accounts for the peak frequency of $\hat{P}_{\alpha}^{y}\left(=\hat{P}_{\alpha}^{y(+)}\right)$ observed for the two-terminal model. Equation (23) is also useful for interpreting the numerical results because it immediately predicts that $\hat{P}_{1}^{y}(\omega, 0)=\hat{P}_{2}^{y}(-\omega, 0)$ and $\hat{P}_{1}^{y}(0, \bar{\omega})=\hat{P}_{2}^{y}(0,-\bar{\omega})$. In addition to these relations, the observation includes the similarity between $\hat{P}_{\alpha}^{y}( \pm v, 0)$ and $\hat{P}_{\alpha}^{y}(0, \mp v)$, implying that $\hat{P}_{\alpha}^{y}(\omega, \bar{\omega})$ is simply a function of the detuning from resonance $\omega-\left(\bar{\omega} \pm \lambda k_{F}\right)$.

Generally, the Rashba SO coupling induces the spin precession at the frequency $\boldsymbol{\omega}_{\mathrm{SO}}=\gamma \mathbf{B}_{\mathrm{SO}}=\lambda \mathbf{k} \times \mathbf{e}_{z}$ in the course of electron transport. It may be convenient here to introduce the precession length $L_{\mathrm{SO}}=v_{F} / \omega_{\mathrm{SO}}=1 / m \lambda$ over which spin precesses by one radian. Note that this definition differs from the conventional one, which considers the precession angle $\pi$, i.e., the evolution of the spin- $\uparrow(\downarrow)$ state to the spin- $\downarrow$ ( $\uparrow$ ) state. ${ }^{27)}$ The electron lifetime $\tau_{F}=L / 2 v_{F}$ in the sample region of size $L$ is used to estimate the mean precession angle at $\theta_{\mathrm{SO}}=\omega_{\mathrm{SO}} \tau_{F}=L / 2 L_{\mathrm{SO}}$. In the two-terminal model assumed for the numerical calculation, the precession occurs predominantly around the $y$-axis and does not oscillate the spin- $S_{y}$ current. Nevertheless, the spin precession is an important concept for understanding the spin pump operation in the finite-sized system. The precession frequency $\omega_{\text {so }}$ coincides 
with the energy separation between the Rashba spin-split subbands. This means that if $\theta_{\mathrm{SO}}>1$, the Rashba energy splitting is well established without being smeared out by the finite-size effect. In such a case, the Kubo formula implies that the driving spin torque for spin pumping diminishes significantly. Figure 5 shows the pumped spin $\hat{P}_{1}^{y}$ calculated as a function of $\lambda$ for $L=55$. When $\theta_{\mathrm{SO}}<1, \hat{P}_{1}^{y}$ shows a quadratic dependence on $\lambda$. This behavior is accounted for by the lowest-order approximation to $J_{\alpha}^{y}$ for a sufficiently small $\lambda$, which predicts that $J_{\alpha}^{y} \propto \lambda^{2}$ through a factor $\tilde{\omega}^{2}$. On the other hand, the increase of $\hat{P}_{1}^{y}$ is appreciably suppressed for $\theta_{\mathrm{SO}}>1$, showing a clear deviation from the $\lambda^{2}$-dependence. The numerical results for different $L$ are similar (not shown). This observation corroborates the interpretation based on the spin torque and the spin precession.

Thus far, we have addressed a clean Rashba SO coupled system in the ballistic transport regime. The remaining part of this paper deals with how the static disorder affects the spin transport and the spin pumping. Since the precession frequency $\omega_{\text {so }}$ is momentum dependent, the successive scattering from impurities randomizes the spin precession process, yielding the so-called D'yakonov-Perel' spin relaxation in two dimensions. ${ }^{27,28)}$ The electron spin rotates by an angle $\phi_{\mathrm{SO}}=\omega_{\mathrm{SO}} \tau$ during the momentum relaxation time $\tau$, leading to the spin relaxation time expressed as $\tau_{\text {spin }}=1 / \omega_{\mathrm{sO}}{ }^{2} \tau$ for $\phi_{\mathrm{SO}}<<1$ in terms of a Brownian rotational diffusion. This expression describes the motional narrowing effect, ${ }^{24)}$ i.e., the frequent impurity scattering slows down the spin relaxation. ${ }^{27,28)}$ The spin diffusion length may be defined as $L_{\text {spin }}=\sqrt{D \tau_{\text {spin }}}$ with $D=\ell^{2} / 2 \tau$ (where $\ell=v_{F} \tau$ is the elastic mean free path), which is almost the same as the precession length $L_{\mathrm{SO}}$. Recall that the 
precession frequency $\omega_{\text {so }}$ coincides with the Rashba energy splitting. This means that if $\phi_{\mathrm{SO}}<1$, the energy splitting is smeared out by the disorder effect. The angle $\phi_{\mathrm{SO}}$ is re-expressed as $\phi_{\mathrm{SO}}=\ell / L_{\mathrm{SO}}$ so that the criterion corresponds to $\ell<L_{\mathrm{SO}}$ in the length scale.

It may be helpful for elucidating the spin transport in the presence of static disorder to employ the spin-resolved transmission coefficients. ${ }^{27)}$ The transmission coefficient from the spin- $\sigma_{v}$ channel in lead $\beta$ to the spin- $\sigma_{\mu}$ channel in lead $\alpha$ is defined by $t_{\alpha \beta}^{\sigma_{\mu} \sigma_{v}}=\operatorname{Tr}\left[\Gamma_{\alpha}^{\sigma_{\mu}} g^{+} \Gamma_{\beta}^{\sigma_{v}} g^{-}\right]$, where $\Gamma_{\alpha}^{\sigma_{\mu}}=p_{\sigma_{\mu}} \Gamma_{\alpha} p_{\sigma_{\mu}} \quad$ and $\quad p_{\sigma_{\mu}}=1 / 2+\sigma_{\mu} S_{\mu} \quad$ is the projection operator onto the eigenstate of spin $S_{\mu}$. The spin-resolved transmission coefficients $t_{\alpha \beta}^{\sigma_{\mu} \sigma_{v}}$ are used to introduce the normalized spin transmittance expressed as

$$
T_{\alpha \beta}^{\mu \nu}=\frac{t_{\alpha \beta}^{\uparrow_{\mu} \uparrow_{v}}+t_{\alpha \beta}^{\downarrow_{\mu} \downarrow_{v}}-t_{\alpha \beta}^{\uparrow_{\mu} \downarrow_{v}}-t_{\alpha \beta}^{\downarrow_{\mu} \uparrow_{v}}}{t_{\alpha \beta}^{\uparrow_{\mu} \uparrow_{v}}+t_{\alpha \beta}^{\downarrow_{\mu} \downarrow_{v}}+t_{\alpha \beta}^{\uparrow_{\mu} \downarrow_{v}}+t_{\alpha \beta}^{\downarrow_{\mu} \uparrow_{v}}}=\frac{4 \operatorname{Tr}\left[S_{\mu} \Gamma_{\alpha} g^{+} S_{v} \Gamma_{\beta} g^{-}\right]}{\operatorname{Tr}\left[\Gamma_{\alpha} g^{+} \Gamma_{\beta} g^{-}\right]},
$$

which describes a correlation between the spin- $S_{\mu}$ current in lead $\alpha$ and the spin- $S_{v}$ current in lead $\beta$, and varies between 1 and -1 , depending on the spin precession and the spin relaxation during the electron transport. Figure 6 summarizes the spin transmittances $T_{12}^{\mu v}$ calculated for the two-terminal systems of the same width $L_{y}=100$ having a disordered and SO coupled region of different length $L_{x}$. The static disorder caused by nonmagnetic impurities is taken into account by introducing a random on-site potential $U(\mathbf{r})$ uniformly distributed in the range $|U(\mathbf{r})| \leq W / 2$. The parameters $W=10$ and $\lambda / 4=0.1$ assumed for the calculation amount to $\ell \cong 0.15$ and $L_{\mathrm{SO}}=5$, respectively, which realize a fully diffusive regime where $\ell<<L_{x}$ and $\ell<<L_{\mathrm{SO}}$. The numerical results collected in the top panel illustrate the spin 
transmittances $T_{12}^{\mu x}(\mu=x, y, z)$. The spin precession during the transport takes place predominantly in the $x z$ plane so that $T_{12}^{x x}$ and $T_{12}^{z x}$ oscillate while $T_{12}^{y x}$ remains around zero. The oscillation gradually decays due to the spin relaxation. Consequently, all the transmittances $T_{12}^{\mu x}$ tend to vanish for a sufficiently large $L_{x}$, indicating that the static disorder significantly deteriorates the spin coherence between two leads. The damped oscillation is reasonably represented by a simple expression, $\cos \left(L_{x} / L_{\mathrm{SO}}+\phi\right) \exp \left(-L / L_{\text {spin }}\right)$, with $\phi=0$ for the diagonal transmittance $T_{12}^{x x}$ and $\phi=\pi / 2$ for the off-diagonal transmittance $T_{12}^{2 x}$, which quantifies the associated spin diffusion length to be $L_{\text {spin }} \cong 5 L_{\mathrm{SO}}$. The observations for $T_{12}^{\mu z}$ (bottom panel) are essentially similar, except for $\phi=-\pi / 2$ for $T_{12}^{x z}$. The absence of an oscillatory behavior for the diagonal transmittance $T_{12}^{y y}$ (middle panel) is simply accounted for by the spin precession around the $y$-axis. A fit to the corresponding numerical result with an exponential function $\exp \left(-L / L_{\text {spin }}\right)$ gives the estimate $L_{\text {spin }} \cong 3 L_{\text {so }}$. These observations demonstrate that the spin diffusion is anisotropic in practice, and is not quantitatively described by the simple relation $L_{\text {spin }} \cong L_{\mathrm{SO}} \cdot{ }^{27)}$ The shorter diffusion length found for $T_{12}^{y y}$ is seemingly problematic for the spin pumping considered in this study since it principally generates the spin- $S_{y}$ current.

Now we proceed to the discussion of the spin pumping in the presence of static disorder. First of all, it should be noticed that the symmetry under the transformation $y \rightarrow-y$ is broken in the presence of the random potential $U(\mathbf{r})$ so that eq. (22) no longer holds. ${ }^{29)}$ For convenience, we here define the magnitude $\hat{P}_{\alpha}=\left|\hat{\mathbf{P}}_{\alpha}\right|$ and the polar angle from the $y$-axis $\theta_{\alpha}=\cos ^{-1} \hat{P}_{\alpha}^{y} / \hat{P}_{\alpha}$ of the pumped spin $\hat{\mathbf{P}}_{\alpha}=\sum_{\mu} \mathbf{e}_{\mu} \hat{P}_{\alpha}^{\mu}$. Figure 7 illustrates the magnitude $\hat{P}_{1}$ and the angle $\theta_{1}$ for the sample-size $L=35$ 
calculated as a function of the disorder strength $W$ for various SO coupling strengths $\lambda$. The magnitude $\hat{P}_{1}$ is little affected by a weak disorder, whereas the broken symmetry produces a finite angle $\theta_{1}$, which increases with $W$ as well as $\lambda$. The magnitude $\hat{P}_{1}$ shows a small step-like increase around the transition between the ballistic transport regime where $\ell>L$ and the diffusive transport regime where $\ell<L$. This behavior is attributable to the disorder-induced smearing of the density of states in quasi-1D systems, since the density of states $N(E)$ for $L=35$ forms a local minimum at $E=0.1$ (as implied from Fig. 3) so that it tends to increase with moderate increases in disorder. A step-like increase is indeed observed for $N(E)$ around $\ell=L$ in the numerical calculation (not shown). In the diffusive regime, the magnitude $\hat{P}_{1}$ increases with $W$ by orders of magnitude for all values of $\lambda$ assumed for the calculation. This increase is too large to be explained by the smeared density-of-states. On the other hand, the angle $\theta_{1}$ tends to saturate in this region. The saturation angle $\theta_{\text {sat }}$ is approximately linear in $\lambda$, and follows the relation that $\theta_{\text {sat }}=(0.2-0.3) \times \theta_{\mathrm{SO}}$. The observations for $\hat{P}_{2}$ and $\theta_{2}$ are similar (not shown). Figure 8 displays a typical example of $\hat{P}_{1}^{y}$ calculated as a function of $\bar{\omega}$ in the presence of disorder. The resonance line clearly exhibits the motional narrowing, indicating that the D'yakonov-Perel' mechanism substantially affects the EDSR-induced spin pumping in the diffusive regime.

As for a weak SO coupling such that $\lambda / 4 \leq 0.01\left(\theta_{\mathrm{SO}} \leq 0.35\right)$, the enhancement of spin pumping is qualitatively explained in terms of a Brownian electron motion in real space, which inevitably prolongs the average time an electron remains in the sample region and is exposed to the pumping field, giving a greater probability of spin 
flip. This interpretation is quantitatively supported by the diagrammatic perturbation analysis in the framework of the weak localization theory. (This argument is based on the identity $J_{\mu}=G_{\mu}$ for $H_{\mathrm{so}}=0$. The weak-localization correction to the spin torque $G_{\mu}$, which can be expressed as a spin-spin correlation function, is discussed in ref. 2.) The enhancement observed for $\lambda / 4=0.1\left(\theta_{\mathrm{SO}}=3.5\right)$ may be largely helped by the disorder-induced broadening that collapses the Rashba energy splitting when $\phi_{\mathrm{SO}}=\ell / L_{\mathrm{SO}}<1$, which corresponds to $W>2$ for $\lambda / 4=0.1$. In addition to the weak localization effect, it is also likely that the motional narrowing assists the enhancement at resonance. A classical treatment may be instructive for considering the saturation angle $\theta_{\text {sat }}$ observed in the diffusive regime. In this regime, an electron remains in the sample region of size $L$ during the time $\tau_{D}=L^{2} / 3 D$. The electron spin follows a random walk and fluctuates around the $y$-axis before escaping into the leads. After the time $\tau_{D}$, the standard deviation of polar angle evolves to $\theta_{\text {spin }} \cong \sqrt{\tau_{D} / \tau_{\text {spin }}}$ by experiencing $\tau_{D} / \tau$ steps of the random walk. This expression can be rewritten as $\theta_{\text {spin }}=L / \sqrt{3} L_{\text {spin }}$. For instance, $L_{\text {spin }} \cong 3 L_{\text {so }}$ evaluated from the spin transmittance $T_{12}^{y y}$ gives $\theta_{\text {spin }} \cong 0.4 \theta_{\text {So }}$, which does not largely differ from the observation for $\theta_{\text {sat }}$. An important implication from this argument is that the pumped spins projected onto the $x z$ plane are essentially random (there is no preferred direction in this plane), and the saturation angle $\theta_{\text {sat }}$ represents the degree of spin dephasing in the diffusive transport regime. A small dephasing is desired for practical application. It is suggested from the numerical results that this is realized by reducing $\theta_{\mathrm{SO}}=L / 2 L_{\mathrm{SO}}$ even for a strong disorder.

Finally, we evaluate the spin current pumped from realistic systems. In order to 
minimize the spin dephasing caused by the disorder, the sample-size $L$ should be sufficiently small. In view of this, we here suppose a sample of size $L=2 L_{\mathrm{SO}}$, for which $\theta_{\text {sat }}=0.2-0.3$. In this case, eq. (24) leads to the spin current expressed as $J_{y}=n \tilde{E}^{2} / 2 m^{2} \omega_{\mathrm{SO}} \omega$ at resonance $\omega=\bar{\omega}$, where $n=N / L^{2}$ is the density of states per unit area. It is implied from this expression that a weaker SO coupling substantially results in a larger spin current. This is due to the constraint on $L$, and opposite to an expectation directly from the pumping field induced by the SO coupling. The SO coupling strengths $\lambda$ have been theoretically and experimentally estimated for some conventional semiconductors. $^{30-33)}$ For example, $\lambda / 2=5 \times 10^{-15} \mathrm{eVm}$ for $\mathrm{Si}$, $10^{-13} \mathrm{eVm}$ for GaAs, and $10^{-11} \mathrm{eVm}$ for InAs, which amount to the precession frequencies, $\omega_{\mathrm{so}} / 2 \pi=0.1 \mathrm{GHz}, 4 \mathrm{GHz}$, and $400 \mathrm{GHz}$, respectively, assuming an electron density of $10^{11} \mathrm{~cm}^{-2}$. Clearly, $\mathrm{Si}$ is a suitable material for the EDSR-induced spin pumping. For Si-based 2D systems, we expect the pumped spin current $J_{y} \cong 10^{13} \mathrm{~s}^{-1}$ for $\omega / 2 \pi=1 \mathrm{GHz}, \bar{B}=36 \mathrm{mT}$, and $\tilde{E}=5 \mathrm{~V} / \mathrm{cm}$. These parameters are chosen to ensure that $\omega_{\mathrm{SO}}<<\omega=\bar{\omega}$ and $\tilde{\omega}<<\bar{\omega}$. It should be noticed that eq. (24) is valid only in the ballistic regime. In the diffusive regime, it is possible that the pumped spin current is increased further.

\section{Conclusions}

The spin pumping from Rashba SO coupled 2D electron systems in the $x y$ plane exploiting the EDSR mechanism has been studied on the basis of the NEGF formalism and the Kubo formalism. An oscillating internal magnetic field in the $x$ direction 
induced by an oscillating external electric field in the $y$ direction through the SO coupling forms the driving spin torque for generating the spin- $S_{y}$ current in the EDSR setup. The Rashba spin-splitting generally tends to diminish the spin torque in infinitely-large clean systems, while this effect becomes less significant for a finite-sized system when the mean precession angle $\theta_{\mathrm{SO}}=L / 2 L_{\mathrm{SO}}$ is smaller than unity. The spin- $S_{y}$ current is pumped without $S_{x, z}$ components from the two-terminal system with a transport channel along $x$ insofar as the system is symmetric under the transformation $y \rightarrow-y$. In the diffusive transport regime, the EDSR-induced spin pumping is strongly enhanced with increasing static disorder. The enhancement is accompanied by motional narrowing as well as spin decoherence due to the D’yakonov-Perel' mechanism. The degree of spin dephasing depends on the electron diffusion time $\tau_{D}$ and the spin dephasing time $\tau_{\text {spin }}$, and is controllable by varying the precession angle $\theta_{\text {so }}$ irrespective of the disorder strength. 


\section{REFERENCES}

1) B. Wang, J. Wang, and H. Guo: Phys. Rev. B 67 (2003) 092408.

2) K. Hattori: Phys. Rev. B 75 (2007) 205302.

3) A. Brataas, Y. Tserkovnyak, G. E. W. Bauer, and B. I. Halperin: Phys. Rev. B 66 (2002) 060404(R).

4) S. Murakami, N. Nagaosa, and S. C. Zhang: Phys. Rev. B 69 (2004) 235206.

5) J. Sinova, D. Culcer, Q. Niu, N. A. Sinitsyn, T. Jungwirth, and A. H. MacDonald: Phys. Rev. Lett. 92 (2004) 126603.

6) P. Sharma and P. W. Brouwer: Phys. Rev. Lett. 91 (2003) 166801.

7) M. Governale, F. Taddei, and R. Fazio: Phys. Rev. B 68 (2003) 155324.

8) C. Li, Y. Yu, Y. Wei, and J. Wang: Phys. Rev. B 75 (2007) 035312.

9) A. G. Mal'shukov, C. S. Tang, C. S. Chu, and K. A. Chao: Phys. Rev. B 68 (2003) 233307.

10) L. Y. Wang, C. S. Tang, and C. S. Chu: Phys. Rev. B 73 (2006) 085304.

11) E. I. Rashba and Al. L. Efros: Phys. Rev. Lett. 91 (2003) 126405.

12) E. I. Rashba and Al. L. Efros: Appl. Phys. Lett. 83 (2003) 5295.

13) Y. Kato, R. C. Myers, D. C. Driscoll, A. C. Gossard, J. Levy, and D. D. Awschalom: Science 299 (2003) 1201.

14) Y. Kato, R. C. Myers, A. C. Gossard, and D. D. Awschalom: Nature (London) 427 (2004) 50.

15) M. Duckheim and D. Loss: Nat. Phys. 2 (2006) 195.

16) V. N. Golovach, M. Borhani, and D. Loss: Phys. Rev. B 74 (2006) 165319. 
17) M. Duckheim and D. Loss: Phys. Rev. B 75 (2007) 201305(R).

18) D. V. Bulaev and D. Loss: Phys. Rev. Lett. 98 (2007) 097202.

19) Y. J. Bao and S. Q. Shen: Phys. Rev. B 76 (2007) 045313.

20) A. P. Jauho, N. S. Wingreen, and Y. Meir: Phys. Rev. B 50 (1994) 5528.

21) S. Datta: Electronic Transport in Mesoscopic Systems (Cambridge University Press, Cambridge, 1995).

22) G. D. Mahan: Many-Particle Physics (Kluwer Academic, Dordrecht/Plenum, New York, 2000).

23) A. Dhar and D. Sen: Phys. Rev. B 73 (2006) 085119.

24) C. P. Slichter: Principles of Magnetic Resonance (Springer-Verlag, New York, 1996).

25) B. Wang, J. Wang, and H. Guo: Phys. Rev. B 68 (2003) 155326.

26) It has been observed in the numerical calculation that including the spin-independent potential $V_{0}(t)$ largely disturbs the spin current pumped from ballistic finite-sized systems, suggesting that in addition to the EDSR mechanism, a kind of parametric quantum spin pumping occurs due to an interplay of the two potentials $V_{0}(t)$ and $V_{\mathrm{SO}}^{( \pm)}(t)$. In the diffusive regime, however, this contribution vanishes while the EDSR effect singly due to the spin-dependent potential $V_{\mathrm{SO}}^{( \pm)}(t)$ survives.

27) T. P. Pareek and P. Bruno: Phys. Rev. B 65 (2002) 241305(R).

28) I. Zutic, J. Fabian, and S. D. Sarma: Rev. Mod. Phys. 76 (2004) 323.

29) It has been confirmed from the additional numerical calculation that the property $\hat{P}_{\alpha}^{x}=\hat{P}_{\alpha}^{z}=0$ is preserved if the disorder potential has the symmetry 
$U(x, y)=U(x,-y)$

30) F. G. Pikus and G. E. Pikus: Phys. Rev. B 51 (1995) 16928.

31) J. P. Heida, B. J. van Wees, J. J. Kuipers, T. M. Klapwijk, and G. Borghs: Phys. Rev. B 57 (1998) 11911.

32) T. Matsuyama, R. Kursten, C. Meibner, and U. Merkt: Phys. Rev. B 61 (2000) 15588.

33) Z. Wilamowski, W. Jantsch, N. Sandersfeld, M. Muhlberger, F. Schaffler, and S. Lyon: Physica E 16 (2003) 111. 


\section{FIGURE CAPTIONS}

Fig. 1

(Color online) Schematic of a setup for EDSR-induced spin pumping from a two-terminal Rashba SO coupled system. The system is exposed to a static magnetic field B. The gates 1 and 2 are used to generate an oscillating electric field $\mathbf{E}(t)$, which produces the internal magnetic field $\tilde{\mathbf{B}}(t)$ through the SO coupling. Spin currents $J_{1,2}^{y}$ pumped at resonance flow into the leads 1 and 2.

Fig. 2

(Color online) Pumped spin $\hat{P}_{\alpha}^{y}(\alpha=1,2)$ calculated as a function of Larmor frequency of static field $\bar{\omega}$ for various sample-sizes $L$. The parameters used in the calculation are indicated in the figure.

Fig. 3

(Color online) Pumped spin $\hat{P}_{\alpha}^{y}(\alpha=1,2)$ at resonance calculated as a function of sample-size $L$. The parameters used in the calculation are indicated in the figure. The dashed line represents the theoretical plot according to eq. (25).

Fig. 4

(Color online) Pumped spin $\hat{P}_{\alpha}^{y}(\alpha=1,2)$ versus Larmor frequency of static field $\bar{\omega}$ (solid line) and pumping frequency $\omega$ (dot) calculated for three different SO coupling strengths: (a) $\lambda / 4=10^{-4}$, (b) $10^{-2}$, and (c) $2 \times 10^{-2}$. The parameters used in the 
calculation are indicated in the figure.

Fig. 5

(Color online) Pumped spin $\hat{P}_{1}^{y}$ calculated as a function of SO coupling strength $\lambda$. The solid line represents the numerical result $\hat{P}_{1}^{y}(0)$ obtained at $\bar{\omega}=0$ while the dot shows the maximum value of $\hat{P}_{1}^{y}(\bar{\omega})$. They follow a $\lambda^{2}$-dependence shown by the dashed line when $\lambda$ is sufficiently small. The parameters used in the calculation are indicated in the figure. The upper horizontal axis is scaled with $L / 2 L_{\mathrm{SO}}$.

Fig. 6

(Color online) Spin transmittances $T_{12}^{\mu v}(\mu, v=x, y, z)$ calculated for two-terminal disordered systems as a function of channel length $L_{x}$. The three panels correspond to (a) $v=x$, (b) $y$, and (c) $z$, respectively. The parameters used in the calculation are indicated in the figure. In the calculation, the disorder average is performed over 1000 random configurations. The upper horizontal axis is scaled with $L_{x} / L_{\mathrm{so}}$.

Fig. 7

(Color online) Pumped spin calculated as a function of disorder strength $W$ for various SO coupling strengths $\lambda$. The two panels show (a) magnitude $\hat{P}_{1}$ and (b) angle $\theta_{1}$. The parameters used in the calculation are indicated in the figure. In the calculation, the disorder average is performed over 1000 random configurations. The upper horizontal axis is scaled with the elastic mean free path $\ell$ estimated from the lowest-order Born approximation as a reference. 
Fig. 8

(Color online) Comparison of resonance lines $\hat{P}_{1}^{y}(\bar{\omega})$ in the presence and absence of static disorder. In the calculation, the disorder average is performed over 1000 random configurations. The parameters used in the calculation are indicated in the figure. 


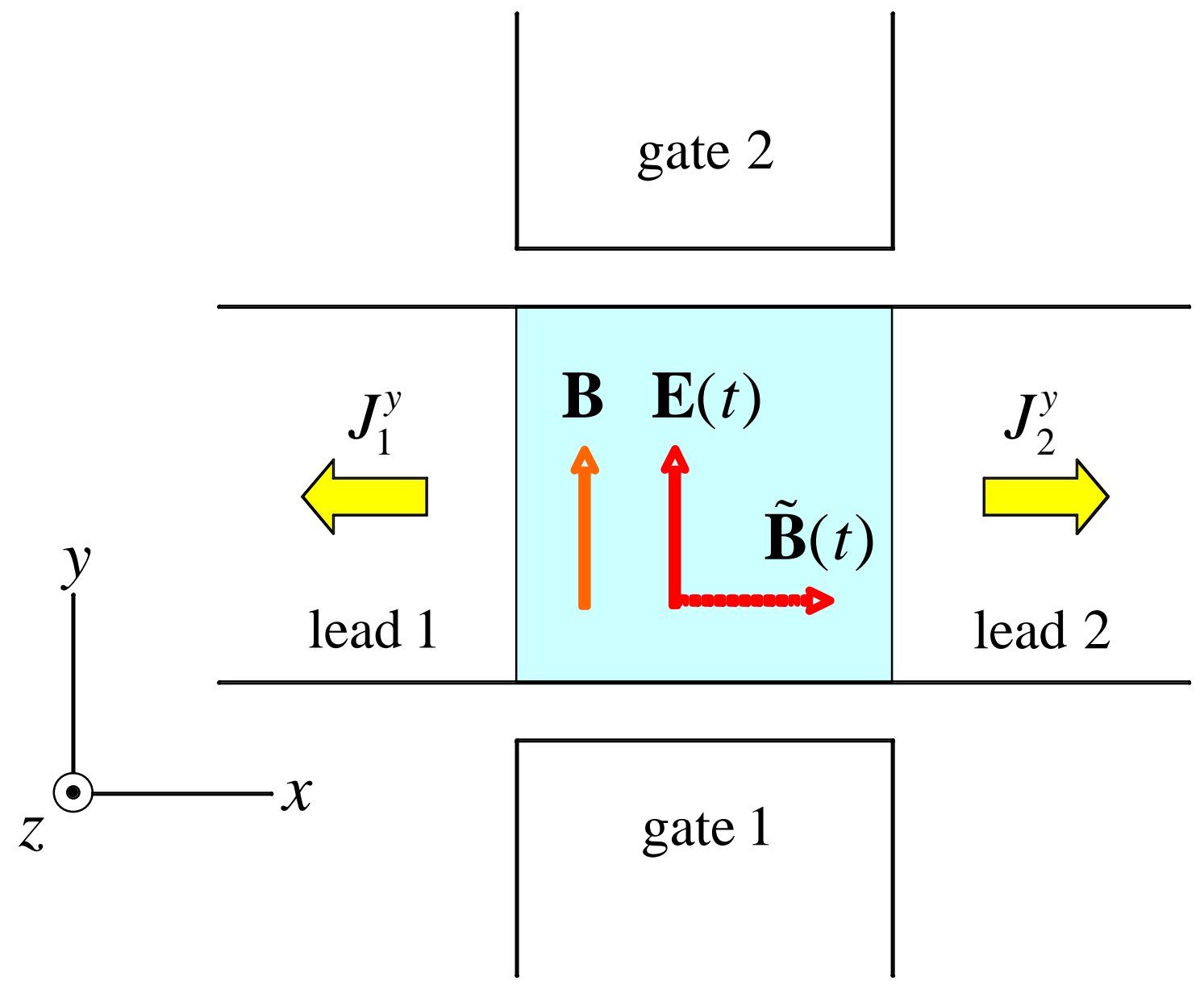

Fig. 1 


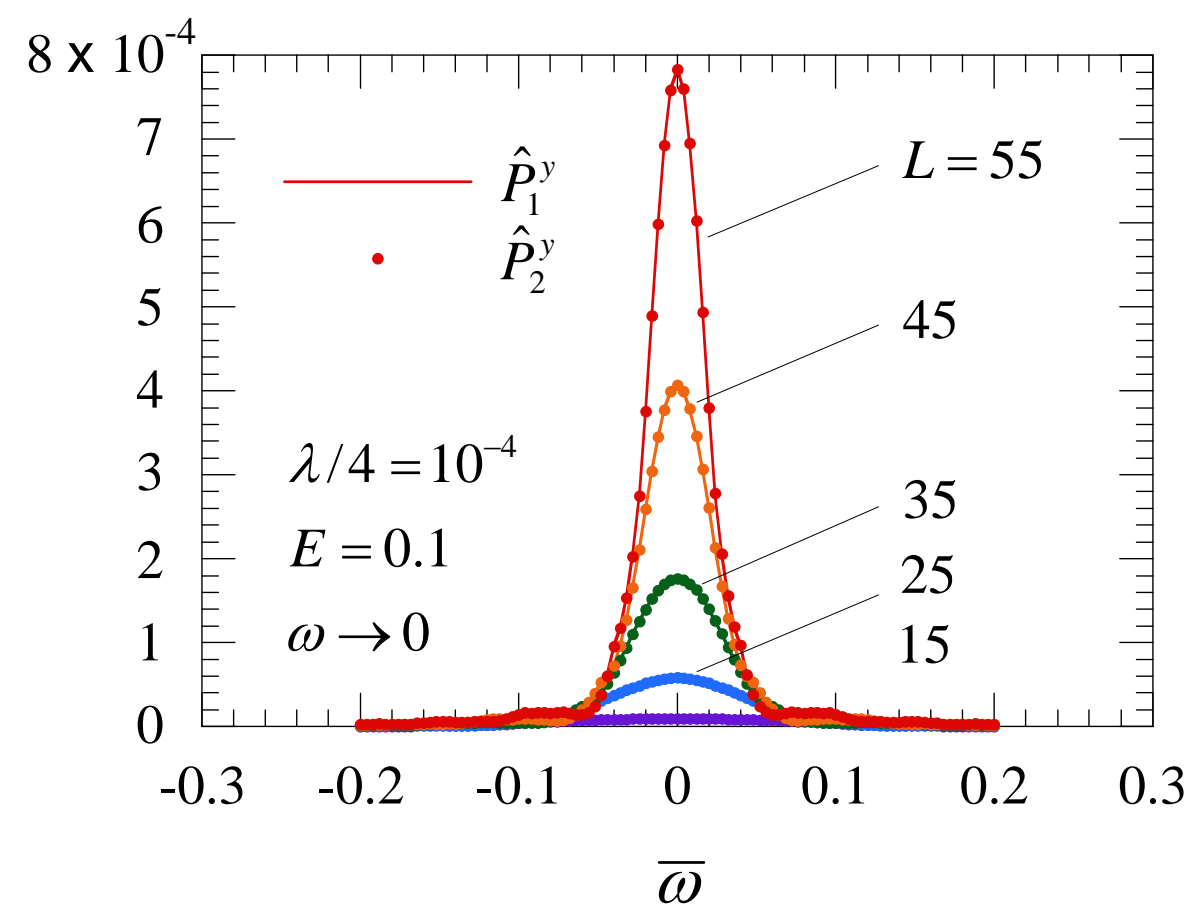

Fig. 2 


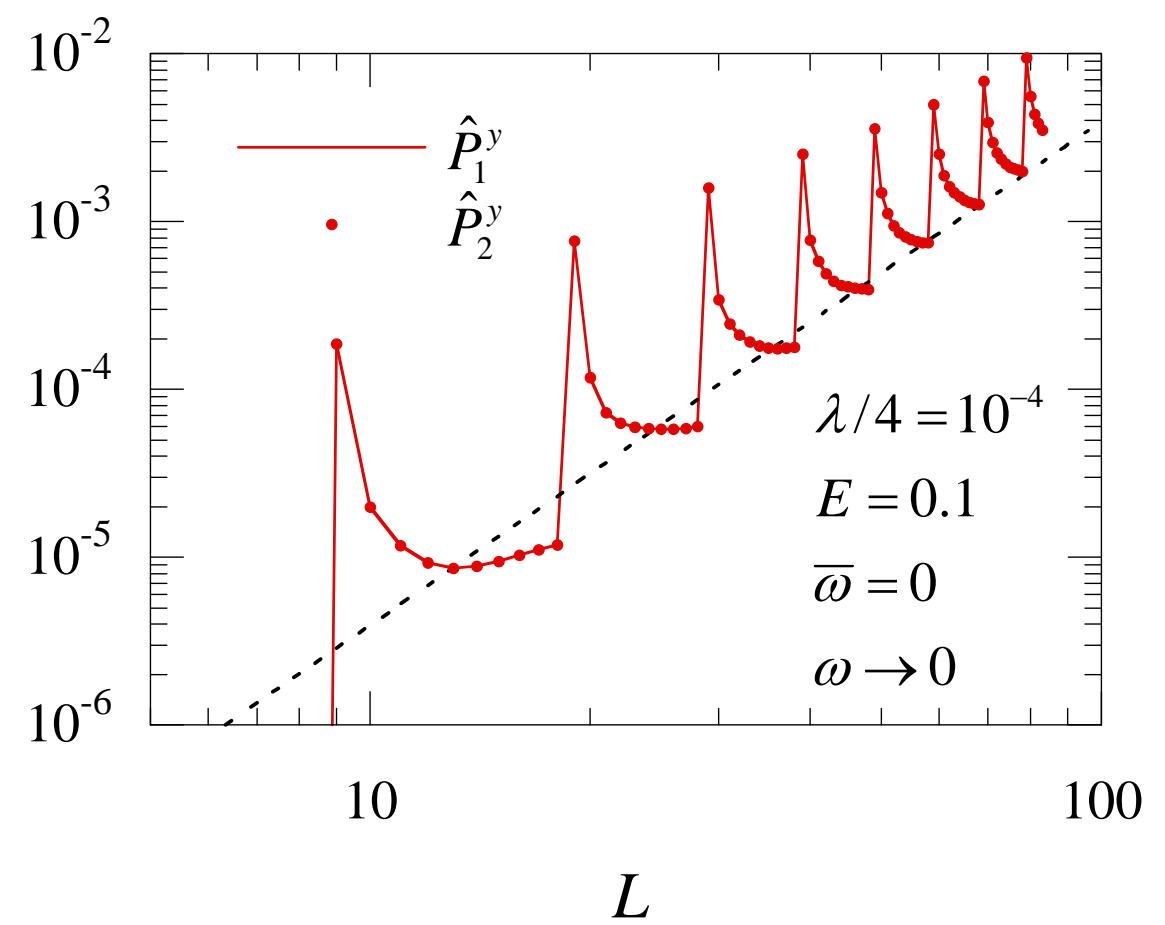

Fig. 3 


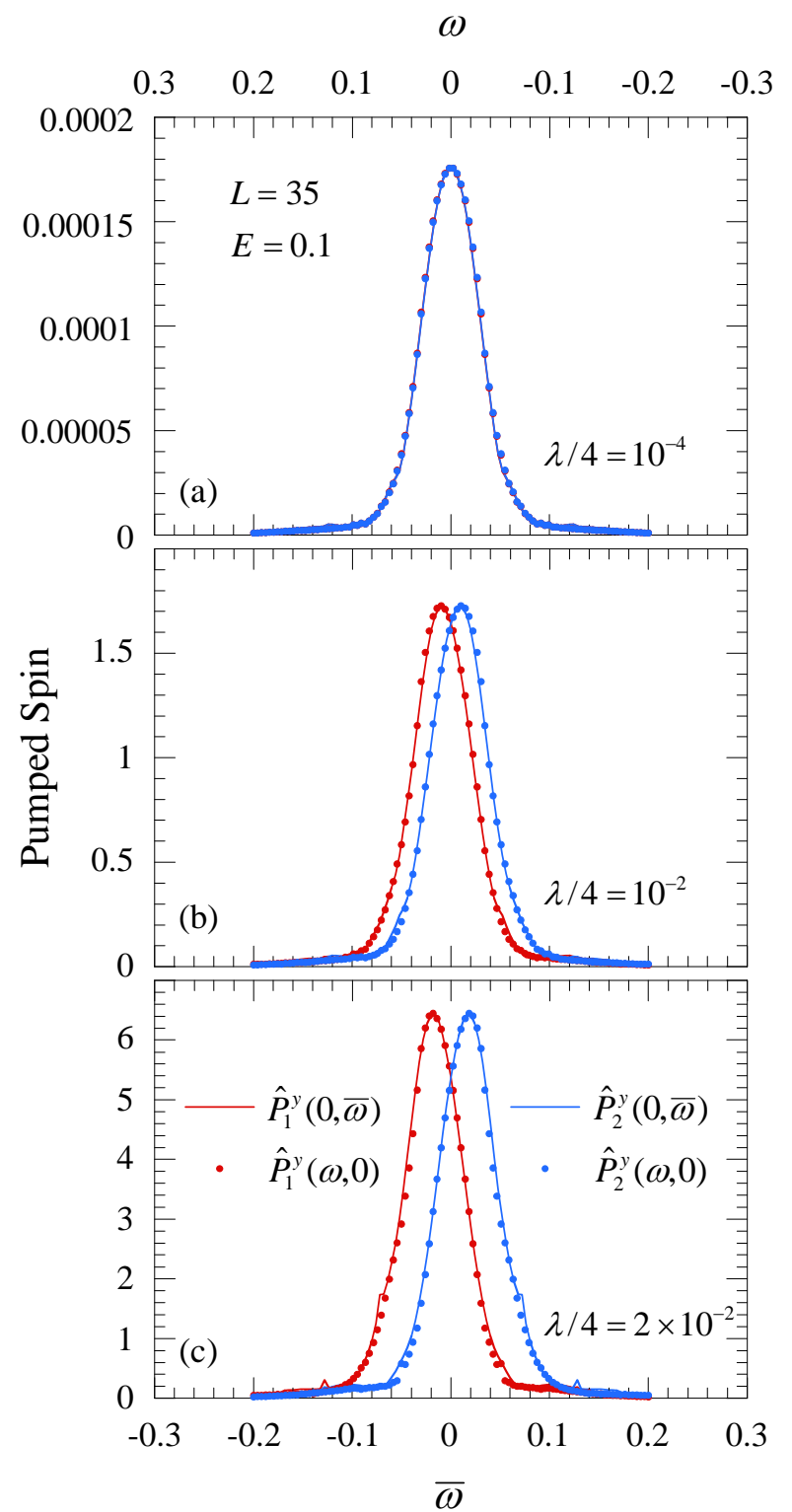

Fig. 4 


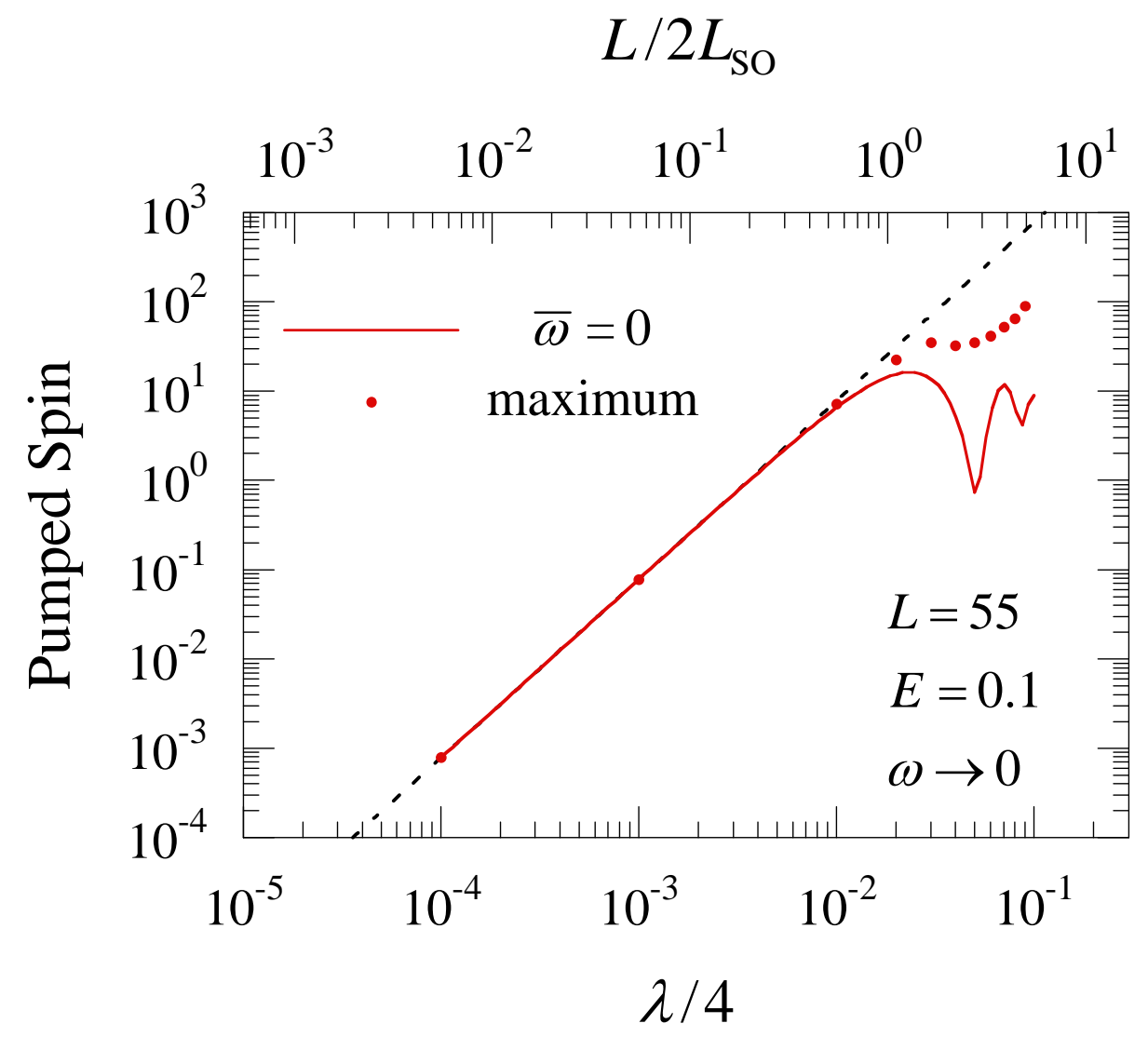

Fig. 5 


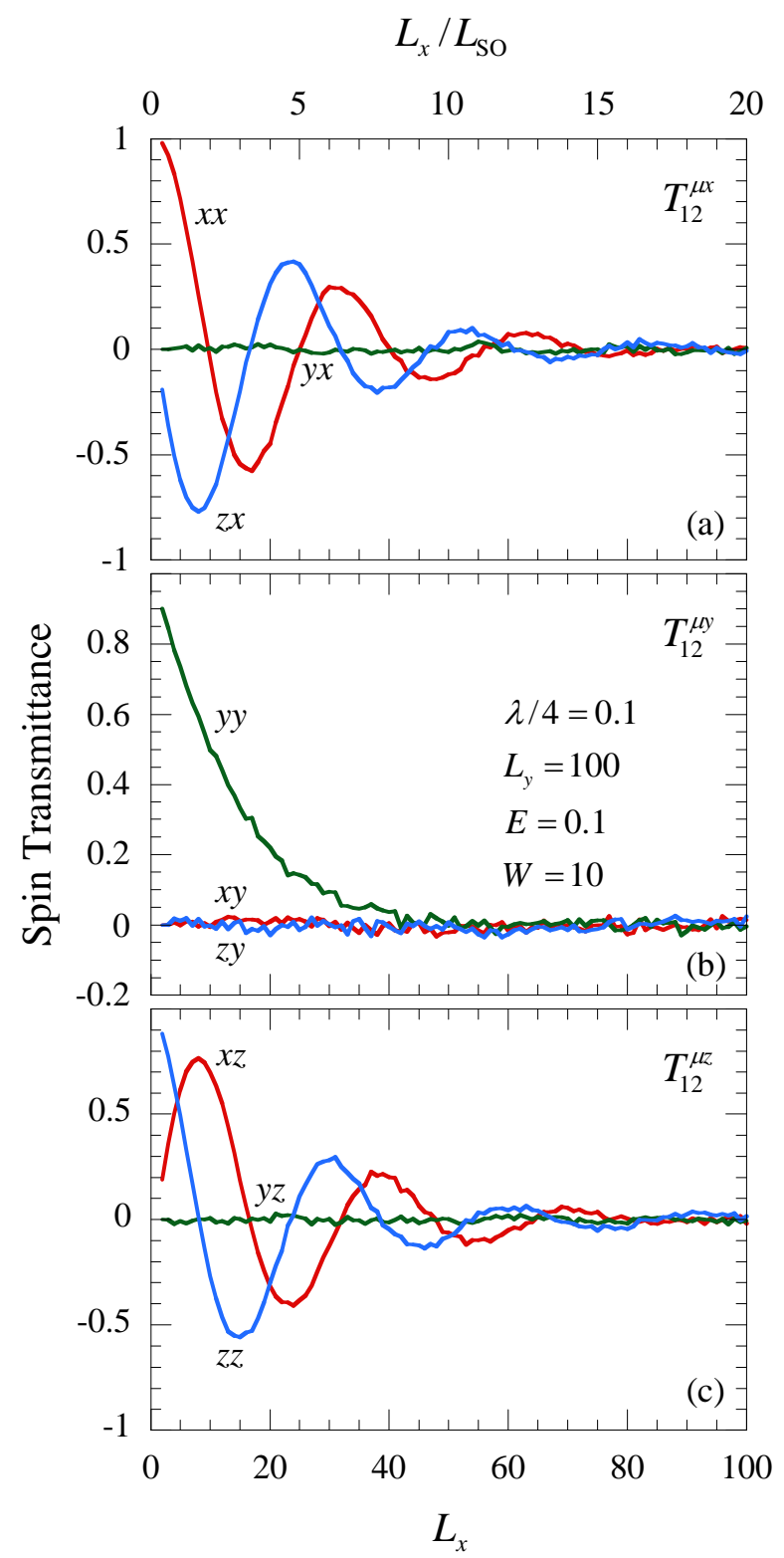

Fig. 6 


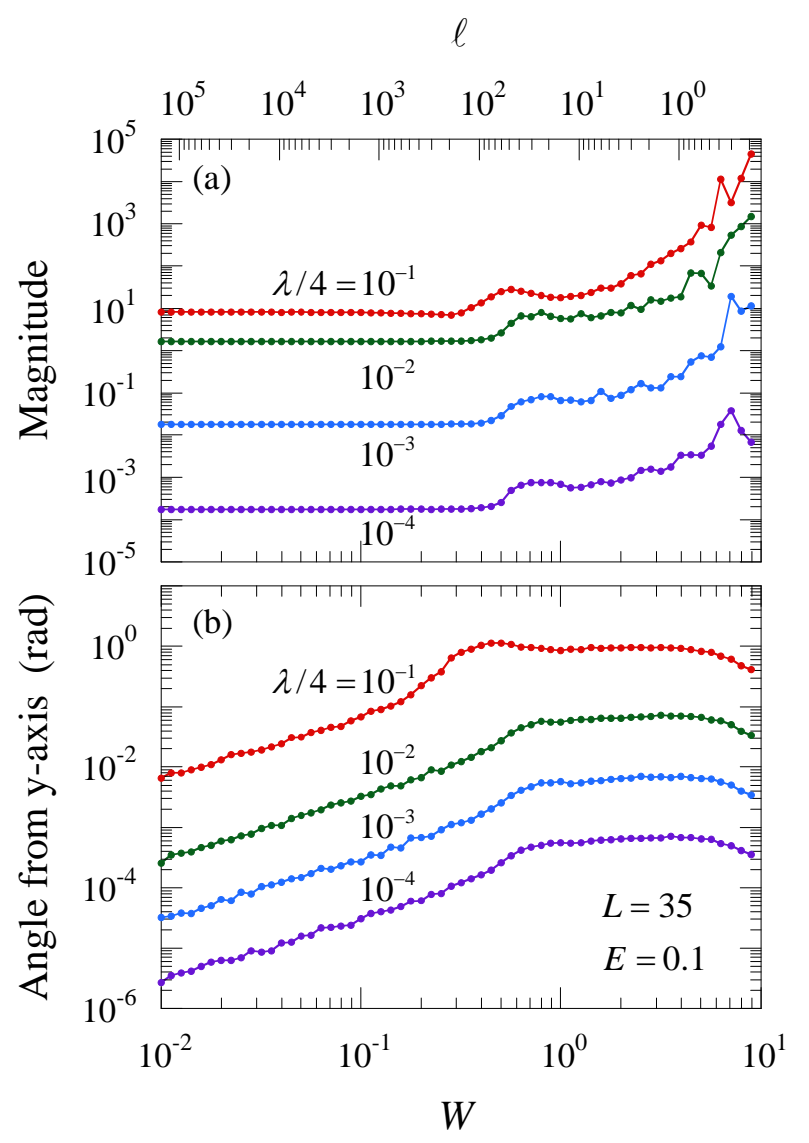

Fig. 7 


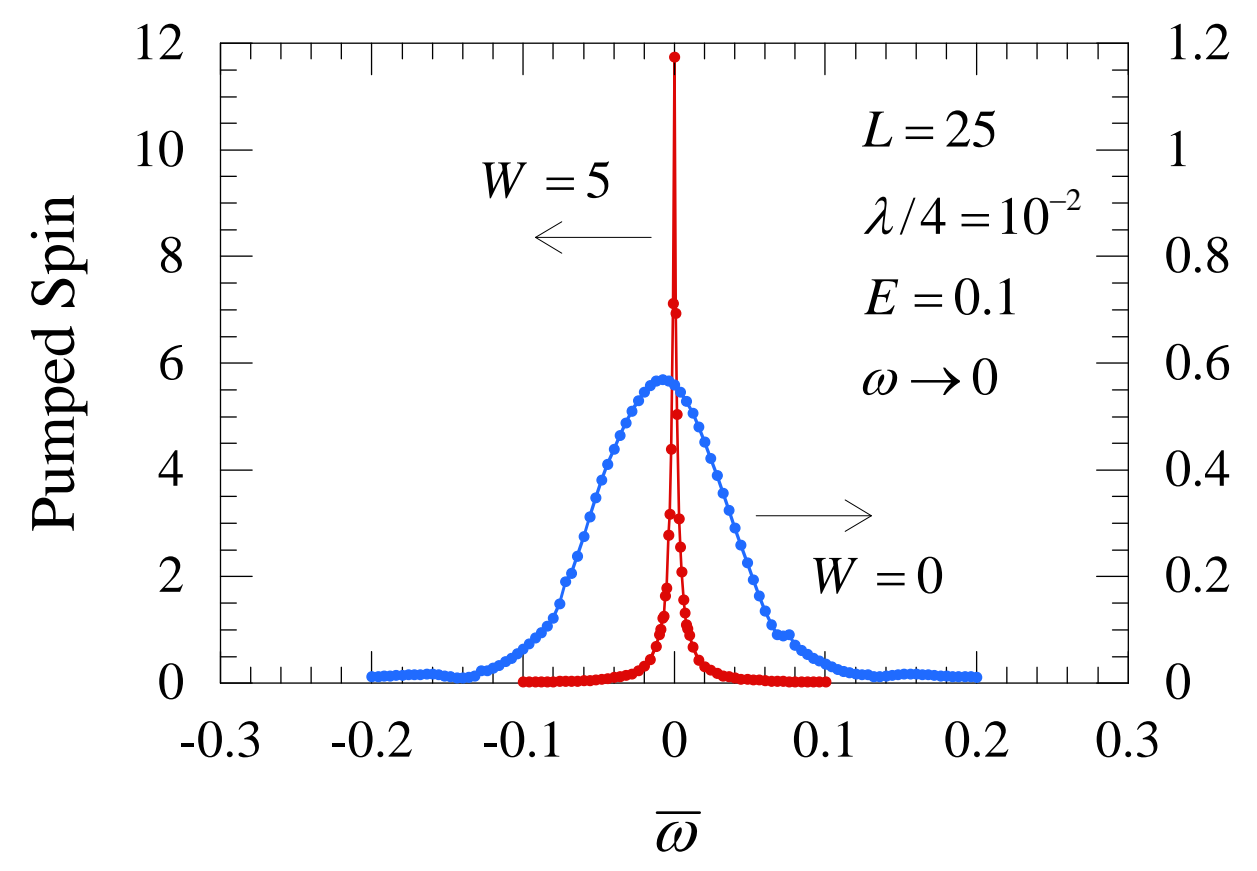

Fig. 8 